\section{New heart rate variability biofeedback}

based on vagal activation: Preliminary CIIS SCALab report 3 ,

A.C. Leterme 1,2 , D. Servant 1,2, Julien De Jonckheere 3, Régis Logier 3, G. Vaiva 1,2

1 Univ. Lille CNRS UMR 9193, SCALab - Sciences Cognitives et Sciences Affectives F-59000 Lille University Hospital, Lille, France.

2 Stress and Anxiety Unit, Department of Psychiatry, Lille University Hospital, Lille, France.

3 Univ. Lille, Inserm, CHU Lille, CIC1403 - Centre d'Investigation Clinique, F-59000 Lille, France.

Background and Aim. Heart rate variability biofeedback (HRV-BFB) involves slowpaced breathing, approximately 6 breaths per minute, which has the effect of maximizing low-frequency heart rate oscillations and exercising the baroreflex. This method may be called low-frequency biofeedback (LF-BFB). Real-time monitoring of vagal activation or parasympathetic nervous system (PNS) activity seems relevant because its measurement can be considered an index associated with stress. Whereas LF-BFB combines the visual tracking of a respiratory sinusal arrhythmia curve with a slow-paced breathing technique, we propose a potential nen complementary biofeedback that couples the visual tracking of vagal activation in real time (the High Frequency Variability Index, HFVI), with the mindful breathing (MB) technique. We call it high-frequency biofeedback (HF-BFB). The purpose of this preliminary report was to describe this novel intervention and to present initial data on patients with anxiety and depressive disorder.

Method. Five patients, 4 men and 1 woman, aged 34 to 59 years old, with stable treatment for at least 3 months, were recruited from the stress and anxiety clinic of Lille University Hospital. They suffered from various anxiety and depressive disorders, such as posttraumatic stress disorder, panic disorder, somatization disorder and major depressive disorder. For each session of HF-BFB, the subject sits on a chair and is fitted with two ECG sensors, one on each wrist, surrounded by a snug elastic strap to prevent signal loss during the exercise. These sensors are connected to the monitoring interface for HF-BFB. The screen of the device faces the subject to allow him to visualize the evolution of his parasympathetic activation. The participant is instructed to follow visually the evolution of the yellow curve, the bar graph and the corresponding number from 0 to 100 and to try to maximize the HFVI score, while he performs an exercise of mindful breathing lasting 10 minutes whose instructions are given on an audio recording.Instantaneous HFVI was recorded just before the onset of the exercise ( $\mathrm{T} 1$ ) five minutes after the onset of the exercise (T2), ten minutes after the onset of the exercise (T3), and five minutes after the end of the exercise (T4). Figure bellow represents the experimental design.

Results. A stimulation of the vagus nerve during HF-BFB for all subjects is highlighted. Indeed, a significant increase from $53 \%$ to $97 \%$ (on a scale of $0 \%$ to $100 \%)$ of parasympathetic activation occurred during this new process. Moreover, even if it was not significant, probably because of the small size of the sample, the parasympathetic activation remained at a higher level five minutes after the end of the exercise $(68 \%)$ than before the exercise $(53 \%)$. Finally, patients reported that this visual feedback of the vagal activation guided them in the practice of this meditation technique, insofar as the variations in the score of parasympathetic activation were related to the degree of involvement in the task, a higher score being associated with attention focused on breathing.

Discussion. These first data support a promising new approach to noninvasively stimulate the vagus nerve and to maintain this effect over time. It could also be a guide allowing to learn more efficiency some stress-reduction techniques requiring a respiratory rate equal to or greater than 9 cycles/minute. This new method could be a complementary approach to LF-BFB, which would diversify the behavioral techniques associated with biofeedback. Controled studies in well-definied population are needed.
A promising new method to stimulate the vagus nerve in a non-invasive way has been developed. This method could be a guide allowing to optimize the learning process of meditation techniques. Biofeedback based on vagal activation could be a complementary approach to the already known respiratory sinus arrhythmia biofeedback.

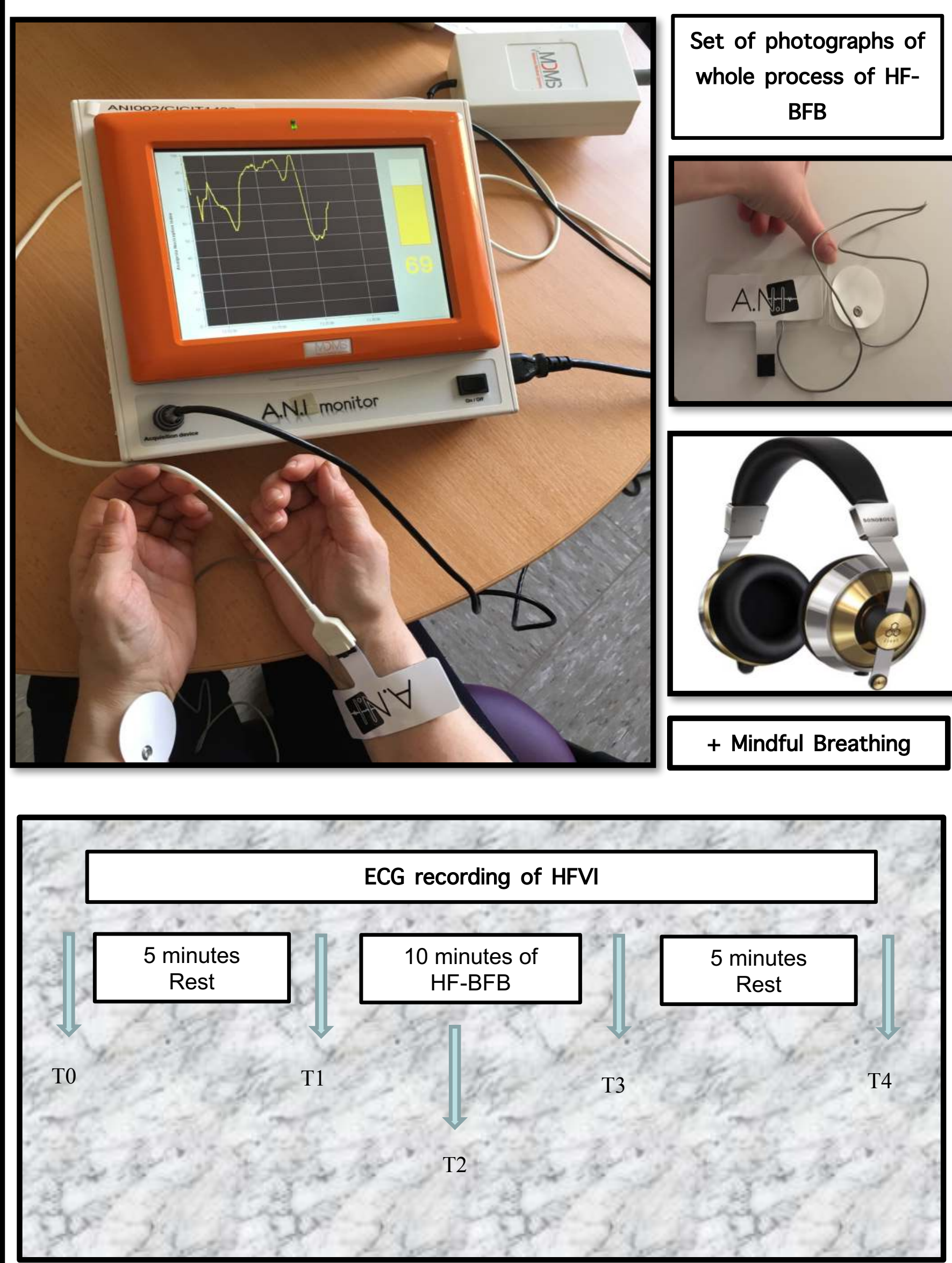

Objectification of vagal stimulation for 5 patients during HF-BFB

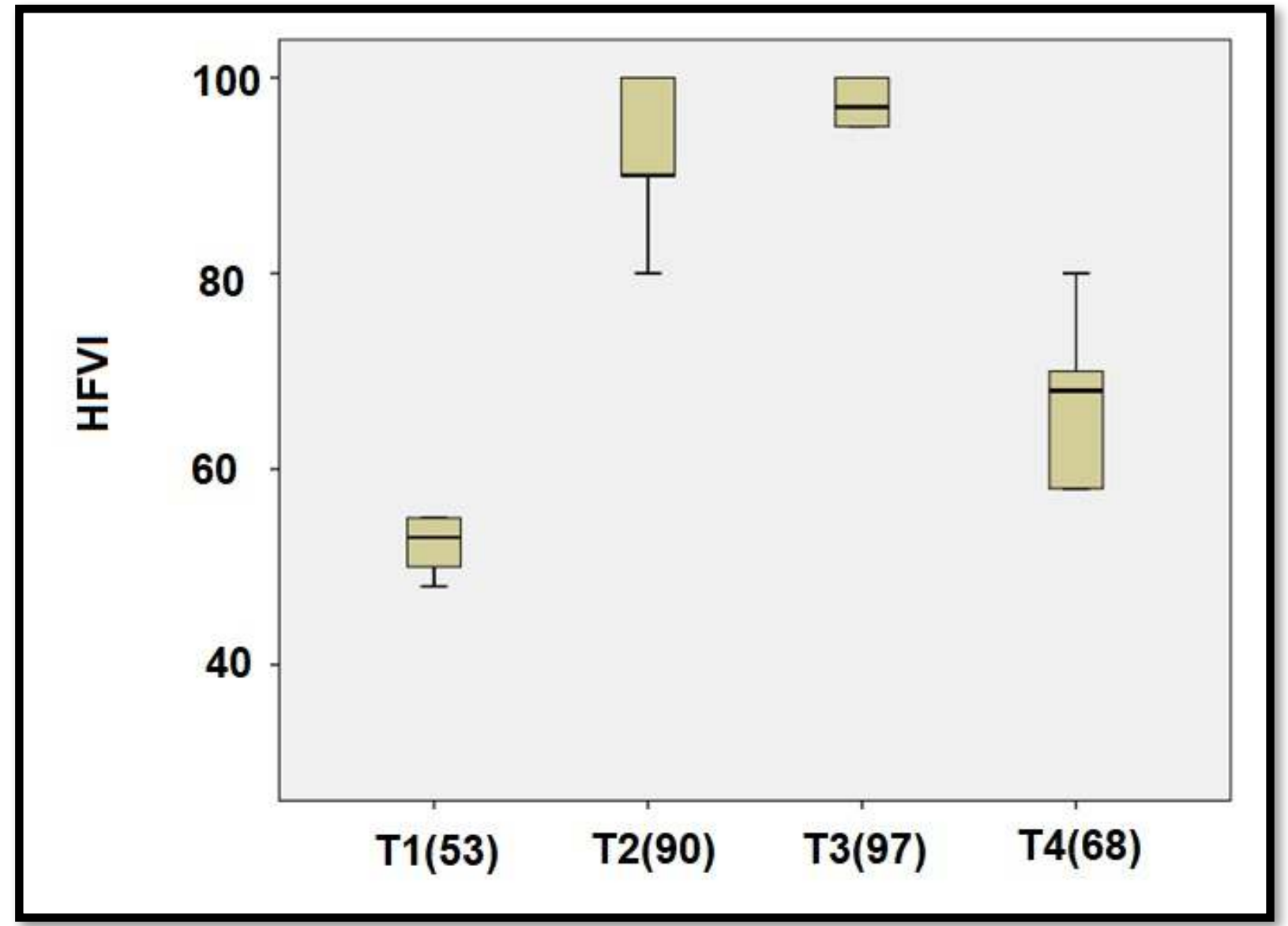

